PHYSICAL REVIEW D 93, 129901(E) (2016)

\title{
Erratum: Lensing in the McVittie metric [Phys. Rev. D 93, 024020 (2016)]
}

\author{
Oliver F. Piattella \\ (Received 26 May 2016; published 9 June 2016)
}

DOI: 10.1103/PhysRevD.93.129901

This erratum is related to Eq. (30) of the original paper. When assuming alignment among observer, lens, and source, we derive the following equation for the deflection angle:

$$
\delta=\frac{4 M\left(1+\chi_{L} H_{0}\right)}{\theta_{S}\left(\chi_{S}-\chi_{L}\right)}+\frac{\chi_{L}}{2\left(\chi_{S}-\chi_{L}\right)}+\mathcal{O}\left(\theta_{S}\right)
$$

Using the angular-diameter distance between lens and source, defined as $D_{L S}=a_{S}\left(\chi_{S}-\chi_{L}\right)$, we write

$$
\theta_{S}\left(\chi_{S}-\chi_{L}\right)=\theta_{S}\left(1+z_{S}\right) D_{L S}=\left(1+z_{S}\right) \theta_{E} D_{L},
$$

and state that the last equality holds true since the proper distances $\theta_{S} D_{L S}$ and $\theta_{E} D_{L}$ are, indeed, the same and equal to the closest approach proper distance.

Our mistake is that the above equality (2) is incorrect. The reason is the following: the angle $\theta_{S}$ is not an observed angle, but it is the angle formed at the source position by the outgoing light ray and the observer-lens-source axis.

Consider the following Gedankenexperiment: imagine a mirror placed at the closest approach distance to the lens which is able to reflect back to the source the outgoing light ray. The latter could then be detected at the source position but with a different angle say $\theta_{S}^{\prime}$ because of the cosmological expansion. The correct equality then is

$$
\theta_{S}^{\prime} D_{L S}=\theta_{E} D_{L}
$$

The right way of dealing with $\theta_{S}\left(\chi_{S}-\chi_{L}\right)$ is also the most natural one:

$$
\theta_{S}\left(\chi_{S}-\chi_{L}\right)=\theta_{E} \chi_{L}=\theta_{E} D_{L}\left(1+z_{L}\right) .
$$

Substituting this result in the lens equation one obtains the standard result, see e.g. [1]:

$$
\theta_{E}^{2}=\frac{4 M D_{L S}}{D_{L} D_{S}}
$$

since the factor $1+z_{L}$ cancels out. No cosmological corrections are present at the leading order and no corrections to the mass estimates are needed.

Therefore, the conclusions of the paper are completely changed: no cosmological effect manifests itself in the lensing phenomenon at the dominant order. Our results are in agreement with those of Refs. [2-4] and with those of Ref. [5], which appeared in the arXiv after the publication of the original paper.

[1] S. Weinberg, Cosmology, (Oxford University Press, New York, 2008).

[2] M. Park, Phys. Rev. D 78, 023014 (2008).

[3] I. B. Khriplovich and A. A. Pomeransky, Int. J. Mod. Phys. D 17, 2255 (2008).

[4] F. Simpson, J. A. Peacock, and A. F. Heavens, Mon. Not. R. Astron. Soc. 402, 2009 (2010).

[5] L. M. Butcher, arXiv:1602.02751. 